\title{
A comparison of the modern Lie scaling method to classical scaling techniques
}

\author{
James Polsinelli and M. Levent Kavvas \\ Department of Civil Engineering, University of California-Davis, Davis, California, USA \\ Correspondence to: James Polsinelli (jfpolsinelli@ucdavis.edu)
}

Received: 3 September 2015 - Published in Hydrol. Earth Syst. Sci. Discuss.: 9 October 2015

Revised: 14 May 2016 - Accepted: 25 May 2016 - Published: 8 July 2016

\begin{abstract}
In the past 2 decades a new modern scaling technique has emerged from the highly developed theory on the Lie group of transformations. This new method has been applied by engineers to several problems in hydrology and hydraulics, including but not limited to overland flow, groundwater dynamics, sediment transport, and open channel hydraulics. This study attempts to clarify the relationship this new technology has with the classical scaling method based on dimensional analysis, non-dimensionalization, and the Vaschy-Buckingham- $\Pi$ theorem. Key points of the Lie group theory, and the application of the Lie scaling transformation, are outlined and a comparison is made with two classical scaling models through two examples: unconfined groundwater flow and contaminant transport. The Lie scaling method produces an invariant scaling transformation of the prototype variables, which ensures the dynamics between the model and prototype systems will be preserved. Lie scaling can also be used to determine the conditions under which a complete model is dynamically, kinematically, and geometrically similar to the prototype phenomenon. Similarities between the Lie and classical scaling methods are explained, and the relative strengths and weaknesses of the techniques are discussed.
\end{abstract}

\section{Introduction}

Scaling is an important tool that is used extensively in engineering, mathematics, and physics. With scaling, conclusions about the dynamics of a system can be based off of the dynamics of another system at a more convenient scale. This is extremely important when it is necessary to understand interactions of systems whose precise governing equations are either unknown or too difficult to work with directly. Scaling can be used to build models of phenomena for study in the laboratory and has important implications in our understanding of very large- and very small-scale phenomena. Examples of scaling in the sciences are numerous. Bluman and Anco (2002) explain that scaling was used to estimate the explosive shockwave of the atomic bomb in the 1940s, Sedov (1993) contains many examples in engineering, in hydrology Dawdy et al. (2012) and Gupta et al. (2010) discuss scaling in flood frequency analysis, and in hydraulics Ercan et al. (2014) use scaling in open channel flow.

The goal of any scaling method is to predict information on one scale from known information on another scale. This is done by scaling relevant variables so that the dynamics of the system remain essentially unchanged. The different scaling procedures each have a way of assigning relationships between the variables being scaled. Three methods will be considered here. The first technique is a classical scaling methodology based on dimensionless groups of variables found through the Vaschy-Buckingham- $\Pi$ theorem. The second technique determines relationships through a scaling transformation of the governing equations for the phenomenon. The third technique is based on a general theory that has characterized all invariant symmetry transformations admitted by a system of equations. The third method is a relatively new addition to modern scaling technology and is known as the Lie scaling method. This new method is applied and compared to results from classical scaling in order to demonstrate its effectiveness and generality as a scaling technique.

The first technique is based on dimensional analysis, and the scaling is usually referred to as dimensional scaling. The scaling relationships are determined based on the dimen- 
sions; e.g., dimensionless groups of variables can be formed and related to ratios between forces, characteristic lengths and times, fluxes, and other factors. There are numerous dimensionless groups of variables that can be formed, and scaling of the system is based on preserving the dimensionless groups. One of the most widely used tools in dimensional scaling is the famous Vaschy-Buckingham- $\Pi$ theorem, explained in detail in Sedov (1993), and a proof of the theorem is given in Bluman and Anco (2002). Many authors have contributed to the present formulation of the theorem widely known through Buckingham (1921). Macagno (1971) discusses precursors and previous formulations of the theorem. Notable precursors are Fourier (2009), Strutt Lord Rayleigh (1877-1878), and Carvallo (1892). An early statement of the theorem can be seen in Vaschy $(1896,1892)$. Two versions of the theorem were developed (likely) independently by Bertrand (1878) and Riabouchinsky (1911, 1915). The theorem establishes the existence, number, and composition of dimensionless groups for a given set of variables. Dimensional scaling has been applied extensively in a wide range of problems and is the primary tool for scaling used by most scientists and engineers.

The second scaling technique relies on the governing equations of the system. In such methods, scaling transformations of the governing equations are required to be invariant in order to preserve the dynamics of the system. An invariant scaling method known as modified inspectional analysis is the basis for physical models known as sandbox models. Modified inspectional analysis is documented in Bear (1972). It is based on forming the scaling schemes for a system of differential equations by enforcing invariance under the transformation for the scaled equations in the interior of the flow domain. The modified inspectional analysis is a variation of the first technique and in application resembles an intuitive version of the Lie scaling technique.

A third scaling method is a special case of a theoretical technique for symmetry analysis of differential equations. The basis for this general theory is the discovery that the set of invariant transformations has a special group structure, known as the Lie group of transformations. This has led investigators to develop an extensive theory completely characterizing all symmetry transformations that hold a system of equations invariant. The Lie scaling methodology uses this extensive mathematical background to provide an approach to obtain a physically based scaling transformation that depends on the system of equations modeling any dynamical process expressed as an initial-boundary value problem.

The Lie group of transformations is well documented in many mathematical sources, and has been applied by several engineers over the last few decades. The theory was originally put forth in Lie (1888). An accessible source of detailed mathematical explanation of the symmetry transformation theory applied to differential systems is Bluman and Anco (2002). Another good applied reference is Olver (1986). The Lie group method is used to investigate the scale invariance of kinematic wave overland flow problem in Haltas and Kavvas (2011b). An algorithm developed for finding symmetry transformations using the group structure has been discussed in Cayar and Kavvas (2009a) and applied by engineers in Cayar and Kavvas (2009b) to find symmetries in a heterogeneous unconfined aquifer problem. Yung et al. (1994) used the Lie group method to classify symmetries in Richard's equation for heterogeneous flow in the vadose zone. Scaling of sediment transport problems using the Lie group method was done by Carr et al. (2015). Lie scaling was applied to a variety of hydrological problems in Haltas and Kavvas (2011a). The scaling behavior and scale invariance conditions of the hydrodynamic processes were investigated by applying the one-parameter Lie scaling transformation directly to the conservation laws in the Reynolds transport theorem framework in Haltas and Ulusoy (2015). The Lie group used here is analogous to the so-called Lie group of one-parameter stretching transformations in Logan (1987, p. 447).

The focus of this study is on developing invariant scaling transformations, but the Lie group method has implications outside of scaling. Indeed, the discovery of any type of invariant symmetry transformation may have serious consequences regarding the understanding of a system of equations. A theorem due to Noether (1918) states that invariance of a system with respect to a symmetry transformation (translational, time, or rotational) is equivalent to a conservation law. Translational invariance corresponds to conservation of linear momentum, rotational to angular momentum, and time invariance to energy conservation. Invariant similarity transformations may also lead to a change in variables which reduce the order of a differential equation. Reduction of order may reduce a partial differential equation to an ordinary differential equation (e.g., the Boltzman transformation may be derived from invariant similarity transformations and can be applied to some nonlinear diffusion equations to reduce them to an ODE - ordinary differential equation).

Application of the Lie group of invariant transformations has a second important implication in scaling. The successful application of a scaling transformation can be classified as one of two types of self similarity. Type- 1 self similarity corresponds to the usual result of a successful dimensional scaling: when a set of dimensionless groups of parameters is found and at least one member of the group can be shown through a limit to be insignificant. This can be stated mathematically as

$\pi=\phi\left(\pi_{1}, \pi_{2}, \ldots, \pi_{k}\right)$,

with at least one dimensionless group, say $\pi_{1}$, satisfying

$\lim _{\pi_{1} \rightarrow 0} \phi\left(\pi_{1}, \pi_{2}, \ldots, \pi_{k}\right)=\phi\left(\pi_{2}, \ldots, \pi_{k}\right) \neq\{0, \infty\}$. 
Type- 2 self similarity is a subset of the case where the limit as $\pi_{1} \rightarrow 0$ results in a limit of 0 or $\infty$ in Eq. (2). In some cases a real $\theta$ may exist so that

$\pi=\pi_{1}^{\theta} \phi\left(\pi_{2}, \ldots, \pi_{k}\right)+o\left(\pi_{1}^{\theta}\right)$.

The exponents must be found by considerations outside of dimensional analysis, or the Lie analysis. These exponents are called anomalous exponents. See Barenblatt (1996) for explanation.

Analyses of type- 1 self similarity in scaling problems are most common in dimensional analysis and have utility since they can result in the reduction of the number of arguments of the problem. Type- 2 self similarity problems have been seen in engineering and statistical mechanics and are promising for solving difficult problems in hydrology and engineering. Examples can be seen in Polsinelli and Kavvas (2016), Gupta and Mesa (2014), and Goldenfeld (1992).

\section{Application of the Lie scaling method}

The Lie scaling method relies on theory developed to characterize all symmetry transformations that leave a system of equations invariant. A transformation $\overline{\mathbf{x}}=\mathbf{X}(\mathbf{x} ; \boldsymbol{\epsilon})$ depends on a vector of parameters $\boldsymbol{\epsilon}$. The transformation leaves a system of equations $\mathbf{F}(\mathbf{x})$ invariant if $\mathbf{F}(\mathbf{x})=\mathbf{F}(\overline{\mathbf{x}})$. The oneparameter group of symmetry transformation will be considered in this study. The Lie scaling method isolates a subgroup of the one-parameter Lie group of point transformations. Theoretically, all members of the Lie group of point transformations can be found using an algorithm, called the Lie algorithm. In the case of scaling transformations, the general form of the transformations is known. Invariance can be enforced by performing a change in variables in the original equations to the scaled variables and requiring that the boundary conditions and governing equations satisfy scaling invariance. It will also be required that all known functions of scaled variables satisfy functional scaling relationships known as self similarity (defined later). An example of the application of this Lie scaling will be given in Sect. 2.2. A brief overview of the theory and the Lie algorithm will be given below.

\subsection{Summary of group symmetry theory}

For a given system of equations $F(\mathbf{x})=0$, the set of oneparameter transformations which leaves $F$ invariant ("are admitted by $F$ " in the parlance) forms the algebraic group structure. The transformations can be expressed using the differentials of the transformation parameter through a Taylor series. In this way the group of transformations can be represented as an infinite series called the Lie series. The Lie series is usually written in terms of differential operators, $X=\xi_{i} \partial / \partial x_{i}$, where the $\xi_{i}(x)$ are the coefficients of the Taylor series. A compact expression is $\bar{x}=e^{\epsilon X} x$.
In general, finding the Lie groups of symmetries admitted by a system of equations involves extending the operator above to include the independent and dependent variables, as well as all derivates up to the differential order of the system. The coefficients of the extended operator can be shown to satisfy an overdetermined system of partial differential equations. In some special cases the system of PDE (partial differential equation) for the coefficients will be linear and homogeneous. Special cases include the wave equation, nonlinear diffusion equations, and the advection-dispersion equations; common equations for phenomena in hydrology and Earth science. The solution to the overdetermined system of PDE allows the transformations admitted by $F(\mathbf{x})=0$ to be explicitly identified.

The main object of study here is the sets of scaling transformations. The general form of a scaling transformation is known: $\bar{x}=e^{\alpha} x, \bar{y}=e^{\beta} y$; i.e., the operators satisfy $X=$ $\alpha x \partial / \partial x+\beta y \partial / \partial y$. Rather than going through the full Lie algorithm, it is sufficient to simply check for invariance of $F(\mathbf{x})=0$ under the scaling transformation. This involves determining whether there is a scaling transformation so that the invariance condition is satisfied simultaneously on the complete system of equations. The scaling coefficients are found in terms of a single scaling base $\epsilon$, taken as the one and only parameter of the transformation.

\subsection{An example on Lie scaling: application to an unconfined aquifer}

As an example, consider a heterogeneous aquifer subject to a flux boundary condition where the initial height of the saturated surface is $h_{\mathrm{o}}$. For simplicity the unconfined groundwater equations will be modeled by the 2-D Dupuit approximation to the 3-D conservation equation and free surface boundary condition. The Dupuit assumption is a good approximation when the slope of the free surface (or the hydraulic gradient) is small. Scaling of the full 3-D system and nonlinear boundary condition is possible, but it is relatively complex.

The Lie scaling transforms the entire system of equations: the governing equations, as well as the boundary and initial conditions. The method begins by gathering all relevant flow or medium variables and scaling them according to the scaling parameter $\epsilon$. In this example,

$$
\begin{aligned}
& \left\{x, y, t, h, h_{\mathrm{o}}, K, S, W, q_{\mathrm{nf}}^{\prime}\right\} \\
& \quad \rightarrow\left\{\epsilon^{\alpha} x, \epsilon^{\beta} y, \epsilon^{\chi} t, \epsilon^{\delta} h, \epsilon^{\iota} h_{\mathrm{o}}, \epsilon^{\phi} K, \epsilon^{\gamma} S, \epsilon^{\eta} W, \epsilon^{\kappa} q_{\mathrm{nf}}^{\prime}\right\} .
\end{aligned}
$$

$S$ is the specific yield, $W$ is the external stress (e.g., pumping, recharge, evapotranspiration), $K$ is the saturated hydraulic conductivity of the aquifer, $q_{\mathrm{nf}}^{\prime}$ is the specific discharge through the saturated thickness of the aquifer (per unit width $\left(\mathrm{L}^{2} \mathrm{~T}^{-1}\right)$ ), and $h$ is the height of the free surface (above a specified datum). The Dupuit approximating equations can 
be written as

$S \frac{\partial h}{\partial t}=\frac{\partial}{\partial x}\left(K h \frac{\partial h}{\partial x}\right)+\frac{\partial}{\partial y}\left(K h \frac{\partial h}{\partial y}\right)+W$.

Applying the scaling transformation and rearranging the Dupuit equations in terms of the scaled variables,

$$
\begin{aligned}
& \epsilon^{\gamma+\delta-\chi} \bar{S} \frac{\partial \bar{h}}{\partial \bar{t}}=\epsilon^{\phi+2 \delta-2 \alpha} \frac{\partial}{\partial \bar{x}}\left(\overline{K h} \frac{\partial \bar{h}}{\partial \bar{x}}\right) \\
& +\epsilon^{\phi+2 \delta-2 \beta} \frac{\partial}{\partial \bar{y}}\left(\overline{K h} \frac{\partial \bar{h}}{\partial \bar{y}}\right)+\epsilon^{\eta} \bar{W} \\
& \epsilon^{\phi+2 \delta-\alpha} \overline{K h} \frac{\partial \bar{h}}{\partial \bar{x}} \bar{n}_{\bar{x}}+\epsilon^{\phi+2 \delta-\beta} \overline{K h} \frac{\partial \bar{h}}{\partial \bar{y}} \bar{n} \bar{y}=\epsilon^{\lambda} \bar{q}^{\prime} \cdot \bar{n}=\epsilon^{\kappa} \bar{q}_{\mathrm{nf}}^{\prime} \\
& \epsilon^{\delta} \bar{h}=\epsilon^{\iota} \bar{h}_{\mathrm{o}} .
\end{aligned}
$$

The scaled flow equations produce conditions on the scaling exponents, the external forcing, and the hydraulic conductivity. The scaling exponents must satisfy the equations

$$
\begin{aligned}
& \gamma+\delta-\chi=\phi+2 \delta-2 \alpha, \phi+2 \delta-2 \alpha=\phi+2 \delta-2 \beta=\eta \\
& \phi+2 \delta-\alpha=\kappa, \quad \iota=\delta .
\end{aligned}
$$

In the event that any of the hydraulic or medium parameters are functions of scaled quantities, those functions must also satisfy scaling relationships. For example, if $K=$ $K(x, y)$, then $K\left(\epsilon^{-\alpha} \bar{x}, \epsilon^{-\beta} \bar{y}\right)=\epsilon^{\phi} \bar{K}$. Functions satisfying this condition are called self similar. Anything that is a function of scaled variables must likewise be self similar in order for invariance to be possible. Hydrologic stresses are a result of flow through the unsaturated zone or pumping; both processes can vary substantially in space and time. In certain flows, density and permeability may be spatially and/or time variable. Viscosity may be a function of temperature, fluid velocity, and space.

In order to relate the Lie scaling technique to a more classical framework, label the prototype system variables with subscript $\mathrm{p}$ and variables in the model system with subscript $\mathrm{m}$. Then $x_{\mathrm{m}}=\epsilon^{\alpha} x_{\mathrm{p}}$ and similarly for the other variables. The Eqs. (8) and (9) can be expressed in terms of the ratios between model and prototype variables:

$\frac{S_{\mathrm{r}} h_{\mathrm{r}}}{t_{\mathrm{r}}}=\frac{K_{\mathrm{r}} h_{\mathrm{r}}^{2}}{x_{\mathrm{r}}^{2}}, \quad x_{\mathrm{r}}=y_{\mathrm{r}}, \quad \frac{h_{\mathrm{r}}^{2}}{x_{\mathrm{r}}^{2}}=\frac{W_{\mathrm{r}}}{K_{\mathrm{r}}}, \frac{h_{\mathrm{r}}^{2}}{x_{\mathrm{r}}}=\frac{q_{\mathrm{r}}^{\prime}}{K_{\mathrm{r}}}$,

$h_{\mathrm{or}}=h_{\mathrm{r}}$.

The saturated hydraulic conductivity depends on properties of the medium and the fluid. It is often related to the permeability of the medium, as well as the density and viscosity of the fluid: $K=\mathrm{k} \rho \mathrm{g} / \mu$. The density, permeability, and viscosity each may vary as functions of space when the hydraulic conductivity varies. Each of these quantities may also vary with scale. The addition of these new variables must be accounted for in the Lie scaling. The appropriate ratios will be added to the list of scaled model-to-prototype quotients: $k_{\mathrm{r}}=k_{\mathrm{m}} / k_{\mathrm{p}}, \rho_{\mathrm{r}}=\rho_{\mathrm{m}} / \rho_{\mathrm{p}}, g_{\mathrm{r}}=g_{\mathrm{m}} / g_{\mathrm{p}}$, and $\mu_{\mathrm{r}}=\mu_{\mathrm{m}} / \mu_{\mathrm{p}}$. Furthermore, $K_{\mathrm{r}}=k_{\mathrm{r}} \rho_{\mathrm{r}} g_{\mathrm{r}} / \mu_{\mathrm{r}}$. It will also be necessary to introduce the pore diameter of the medium $d$, and the ratio $d_{\mathrm{r}}=d_{\mathrm{m}} / d_{\mathrm{p}}$. Gravity will be assumed constant in space and independent of scale, $g_{\mathrm{r}} \equiv 1$. The permeability is related to the square of the pore diameter through a dimensionless quantity called the Darcy number (Vafai, 2005). Referring to these relations, the first equation in Eq. (10) can be re-written as

$\frac{\left(S_{\mathrm{r}} x_{\mathrm{r}} / t_{\mathrm{r}}\right)^{2}}{g_{\mathrm{r}} h_{\mathrm{r}}} \frac{d_{\mathrm{r}}^{2}}{k_{\mathrm{r}}} \frac{x_{\mathrm{r}}^{2}}{d_{\mathrm{r}}^{2}}=\frac{\left(S_{\mathrm{r}} x_{\mathrm{r}} / t_{\mathrm{r}}\right) \rho_{\mathrm{r}} x_{\mathrm{r}}}{\mu_{\mathrm{r}}}$.

Notice that the quantity on the right resembles the Reynolds number ratio with characteristic velocity $V_{\mathrm{r}}=S_{\mathrm{r}} x_{\mathrm{r}} / t_{\mathrm{r}}$, which is a measure of the horizontal fluid velocity through the pore space; the characteristic length ratio is $x_{\mathrm{r}}$. The quantities on the left include the inverse of the dimensionless Darcy number, defined as the ratio of the permeability to the crosssectional pore area. Also present is the ratio of the crosssectional pore area to the horizontal area of the flow domain, and the ratio between the height of the phreatic surface and the horizontal length of the domain. On the left-hand side of the equation is a quantity that resembles the square of the Froude number ratio with characteristic velocity ratio the same as the right-hand side and characteristic length as the horizontal aspect ratio. This indicates the conditions under which dynamic and kinematic similarity are simultaneously preserved.

The third equation in Eq. (10) can be re-written as

$\frac{\rho_{\mathrm{r}} W_{\mathrm{r}} \mathrm{h}_{\mathrm{r}}}{\mu_{\mathrm{r}}} \frac{k_{\mathrm{r}}}{d_{\mathrm{r}}^{2}} \frac{d_{\mathrm{r}}^{2}}{x_{\mathrm{r}}^{2}}=\frac{W_{\mathrm{r}}^{2}}{g_{\mathrm{r}} h_{\mathrm{r}}}$.

Once again, the quantity on the left is related to the Reynolds number with velocity $W$ oriented in the vertical direction either in or out of the aquifer, depending on the specific conditions and characteristic length proportional to the height of the phreatic surface, $h$. On the right, the dimensionless quantity is related to the square of the Froude number.

The fourth equation in Eq. (10) can be manipulated similarly; here, $q^{\prime}$ is the discharge per unit width. The characteristic velocity is related to the discharge, or specific discharge at the boundary.

$\frac{\left(q_{\mathrm{r}}^{\prime} / h_{\mathrm{r}}\right) \rho_{\mathrm{r}} x_{\mathrm{r}}}{\mu_{\mathrm{r}}} \frac{k_{\mathrm{r}}}{d_{\mathrm{r}}^{2}} \frac{d_{\mathrm{r}}^{2}}{x_{\mathrm{r}}^{2}} \frac{h_{\mathrm{r}}}{x_{\mathrm{r}}}=\frac{q_{\mathrm{r}}^{2} / h_{\mathrm{r}}^{2}}{g_{\mathrm{r}} x_{\mathrm{r}}}$

The characteristic velocity is the specific discharge over the saturated height. The Reynolds number can be seen on the left-hand side and the square of the Froude number on the right-hand side, and the characteristic lengths will be proportional to the horizontal dimensions of the aquifer. The ratios between the pore area and the aquifer area, and the saturated height to the horizontal extent, serve as relations between the dynamic quantities. 
As may be seen above, the Lie scaling approach provides a grounded method for determining scaling conditions for invariance of a set of equations. Scale invariance conditions may be phrased in terms of such quantities as the Reynolds number, Froude number, and other useful non-dimensional properties. This is useful when considering design specifications of experiments and the nature of the forces that are preserved after transformation to the scaled system.

Equations (11), (12), and (13) give explicit relationships between the ratios of important dimensionless quantities in hydrology and fluid dynamics. It allows investigators to specify conditions in terms of the scaling of the flow domain $\left(x_{\mathrm{r}}\right.$, $\left.y_{\mathrm{r}}\right)$, medium properties $\left(k_{\mathrm{r}}\right)$, fluid properties $\left(\rho_{\mathrm{r}}, \mu_{\mathrm{r}}\right)$, and external forcing $\left(W_{\mathrm{r}}, q_{\mathrm{r}}^{\prime}\right)$. The scaling of these variables may be chosen so that the system is both invariant and dynamically similar, e.g., $R e_{\mathrm{r}}=1$ and $F r_{\mathrm{r}}=1$. Under the constraints of invariance and dynamic similarity, the scalings of the system (11), (12), and (13) must satisfy

$$
\begin{array}{ll}
W_{\mathrm{r}}=\left(\frac{\mu_{\mathrm{r}} g_{\mathrm{r}}}{\rho_{\mathrm{r}}}\right)^{\frac{1}{3}} \quad \text { from (12), } \\
S_{\mathrm{r}}=\frac{t_{\mathrm{r}} \sqrt{g_{\mathrm{r}} h_{\mathrm{r}}}}{x_{\mathrm{r}}}, \quad h_{\mathrm{r}}=\left(\frac{\mu_{\mathrm{r}}}{\rho_{\mathrm{r}} x_{\mathrm{r}}}\right)^{2} \frac{1}{g_{\mathrm{r}}} \quad \text { from (11), and } \\
q_{\mathrm{r}}^{\prime}=h_{\mathrm{r}} \sqrt{g_{\mathrm{r}} x_{\mathrm{r}}}, \quad x_{\mathrm{r}}=\left(\frac{\mu_{\mathrm{r}}}{\rho_{\mathrm{r}}}\right)^{\frac{2}{3}}\left(\frac{1}{g_{\mathrm{r}}}\right)^{\frac{1}{3}} \quad \text { from (13). }
\end{array}
$$

From Eqs. (11), (12), and (13) the non-Froude, Reynolds terms require that $k_{\mathrm{r}}=x_{\mathrm{r}}^{2}$, which can be stated as $D a_{\mathrm{r}}=$ $x_{\mathrm{r}}^{2} / d_{\mathrm{r}}^{2} . D a_{\mathrm{r}}$ is the ratio of the Darcy numbers for the model and prototype. Combining relevant quantities in Eqs. (14), (15), and (16) gives

$$
\begin{aligned}
& h_{\mathrm{r}}=x_{\mathrm{r}}=\left(\frac{\mu_{\mathrm{r}}}{\rho_{\mathrm{r}}}\right)^{\frac{2}{3}}\left(\frac{1}{g_{\mathrm{r}}}\right)^{\frac{1}{3}}, S_{\mathrm{r}}=t_{\mathrm{r}}\left(\frac{g_{\mathrm{r}}^{2} \rho_{\mathrm{r}}}{\mu_{\mathrm{r}}}\right)^{\frac{1}{3}}, q_{\mathrm{r}}^{\prime}=\frac{\mu_{\mathrm{r}}}{\rho_{\mathrm{r}}}, \\
& W_{\mathrm{r}}=\left(\frac{g_{\mathrm{r}} \mu_{\mathrm{r}}}{\rho_{\mathrm{r}}}\right)^{\frac{1}{3}} .
\end{aligned}
$$

This scaling scheme is trivial if $g_{\mathrm{r}}=\mu_{\mathrm{r}}=\rho_{\mathrm{r}}=t_{\mathrm{r}}=1$. For most practical problems on Earth, $g_{\mathrm{r}} \equiv 1$. The kinematic viscosity can be modified in an experimental setting. In problems where the Darcy scale equations are upscaled to field, watershed, or regional scales, the question is whether the viscosity of the fluid changes as the scale increases.

The procedure above was structured to preserve two wellknown non-dimensional fluid groups as they are formed in the preceding analysis. Since $x_{\mathrm{r}}=y_{\mathrm{r}}$, geometric similarity will be preserved. Preserving both kinematic and dynamic non-dimensional groups is often difficult and is not always possible. In many problems preserving both dimensionless quantities may not be important. Whether they are held invariant or not, however, the two numbers are linked though Eqs. (11), (12), and (13). This relationship may be referenced to understand the effect of a scaling scheme on the kinematic and dynamic similarity. It may be desirable for investigators to take either $F r$ or $R e$ as design parameters in creating physical models or in upscaling the point scale equations, and to observe the effect of scaling in the chosen variable on the unchosen non-dimensional group.

Other formulations may be of interest in terms of different characteristic velocities and lengths. There are other dimensionless groups that are used in fluid dynamics through porous media; one such number is the Péclet number, defined as the ratio of advective transfer rate to diffusive transfer rate. Investigation of the scaling implications in terms of the Péclet number could prove most interesting for subsurface problems.

\section{Classical models and dimensional scaling}

Dimensional scaling is based on the idea that nondimensional groups can be formed based on the dimensions of the quantities involved in a physical phenomenon. Quantities are usually spatial and temporal lengths, areas, volumes, velocities, forces, resistances/conductances, densities, etc. If the dominant forces and the quantities that the forces act on are known, then dimensionless groups are formed using those forces and associated characteristic properties. This idea is formalized with the famous Buckingham $\Pi$ theorem which operates under assumptions that are true of any physical problem (Bluman and Anco, 2002).

Dimensional analysis in fluid dynamics usually resolves to requiring geometric, kinematic, and/or dynamic similarity. Geometric similarity requires that all body/domain dimensions have the same linear scale ratio. Kinematic similarity requires that the velocity scale ratios are identical. Dynamic similarity requires that the force scale, or mass scale, ratio be the same between the model and prototype. White (2011) summarizes similarity scaling for incompressible flow according to the presence or absence of a free surface. For problems with no free surface, dynamic similarity requires equality in the Reynolds numbers between the model and prototype. In problems with a free surface, the model and prototype Reynolds and Froude numbers must be equal at least. In some cases the Webber and Euler numbers (inertial to surface tension and pressure to inertia, respectively) must be equal as well.

As an example, consider flow in a confined aquifer. Under usual conditions the flow velocity is quite low, so advective effects are reduced and the viscous effects dominate the problem. In this case, there is no free surface and the most important dimensionless quantity is usually taken to be the Reynolds number, defined as the ratio of the inertial forces to the viscous forces. Under the condition that the dynamics of the model be the same as the dynamics of the prototype, the ratio of the Reynolds numbers should be the same.

$\frac{V_{\mathrm{m}} l_{\mathrm{m}} \rho_{\mathrm{m}}}{\mu_{\mathrm{m}}}=\frac{V_{\mathrm{p}} l_{\mathrm{p}} \rho_{\mathrm{p}}}{\mu_{\mathrm{p}}}$.

(dynamic similarity) 
In this dimensional analysis, the values are assigned to $V_{\mathrm{m}}$, $V_{\mathrm{p}}, l_{\mathrm{m}}, l_{\mathrm{p}}, \rho_{\mathrm{m}}, \rho_{\mathrm{p}}, \mu_{\mathrm{m}}$, and $\mu_{\mathrm{p}}$, which are thought to be characteristic or representative of the system. Choosing appropriate values is at the heart of the dimensional analysis scaling problem. The choices that are made often reflect quantities that are measured or observed.

For example, in a hydrologic study modeling the fate of water infiltrated into a hillslope during a storm event, the rainfall rate at the surface can be measured, and the discharge through a seepage face can be measured. These two values give indications of the characteristic velocity of the water in the subsurface. The characteristic lengths will depend on the process. For the vertical infiltration through the hillslope, the characteristic length may be the depth from the surface to the water table. Once the water reaches the saturated zone, the nature of its movement changes from being primarily vertical flow to horizontal flow. For flow in the saturated zone the characteristic length will be related to the horizontal extent of the aquifer.

As a second example consider flow of water through an open channel. Flows like these have relatively high velocities and large Reynolds numbers, and the dimensionless quantity thought to be of greatest importance is known as the Froude number. The Froude number is the ratio of the inertial forces to the gravity forces. Scaling using this ratio proceeds by equating

$$
\frac{V_{\mathrm{m}}}{\sqrt{g_{\mathrm{m}} l_{\mathrm{m}}}}=\frac{V_{\mathrm{p}}}{\sqrt{g_{\mathrm{p}} l_{\mathrm{p}}}} .
$$

(kinematic similarity)

The characteristic velocities are usually taken to be the average velocity of the flow in the channel and the length is usually taken to be the depth of the channel. For closed channels the characteristic length is the hydraulic diameter of the channel.

For the problem in Sect. 2.2, since the aquifer is unconfined there will be a free surface and flow is relatively slow. In this case, the notion of dynamic similarity may need to be modified to include equality of the relevant Froude number as well as the Reynolds number; i.e., kinematic similarity and dynamic similarity must be satisfied simultaneously.

In typical problems, scaling is done either by dynamic similarity or kinematic similarity. It is not usual for equality of both quantities to be satisfied simultaneously. Generally, information relating the various dimensionless quantities is not available, so the scaling of spatial and dynamic quantities cannot be easily done in such a way as to preserve both kinematic and dynamic similarity.

In the scaling problem for unconfined aquifer flow, the condition that the Reynolds numbers between the model and prototype be the same is a condition of the scaling scheme in Eq. (17) derived from conditions in Eqs. (11), (12), and (13). This highlights the fact that the constraints of classical scaling by equating the model and prototype ratios of certain non-dimensional variables may be added as conditions in the
Lie scaling methodology. The question that must be investigated in any Lie scaling application is whether a non-trivial scaling is possible under both the conditions of invariance of the governing equations and boundary conditions, and dynamical/kinematic similarity. The dimensional analysis using only the Reynolds number similarity will allow investigators a high degree of freedom in model design while potentially sacrificing kinematic similarity as well as invariance of the governing equations.

A second established approach for scaling is called modified inspectional analysis and is documented in Bear (1972, chap. 11). In this reference, the sandbox model is based upon modified inspectional analysis. The sandbox model is a scaled physical model of a subsurface zone. It consists of a rigid watertight container filled with a porous matrix. The materials which make up the porous matrix are determined by the designer and the desired scaling properties. Materials can be sand, glass beads, crushed glass, etc.

The scaling procedure is very similar to the Lie scaling technique. Application of the modified inspectional analysis to the groundwater problem in Sect. 2.2 begins with gathering the twelve independent variables: $x, y, t, h, k, \mu, \rho$, $q_{x}, q_{y}, S, W$, and $A$, where $A$ is the horizontal area and $\mathbf{q}$ is the Darcy flux. The variables are scaled and the relationships between the model-prototype ratios are found according to Eq. (4):

$$
\begin{aligned}
& W_{\mathrm{r}}=\frac{k_{x \mathrm{r}} \rho_{\mathrm{r}} h_{\mathrm{r}}^{2}}{x_{\mathrm{r}}^{2} \mu_{\mathrm{r}}}=\frac{k_{y \mathrm{r}} \rho_{\mathrm{r}} h_{\mathrm{r}}^{2}}{y_{\mathrm{r}}^{2} \mu_{\mathrm{r}}}=\frac{S_{\mathrm{r}} h_{\mathrm{r}}}{t_{\mathrm{r}}}, \\
& q_{x \mathrm{r}}=\frac{k_{x \mathrm{r}} \rho_{\mathrm{r}} h_{\mathrm{r}}}{\mu_{\mathrm{r}} x_{\mathrm{r}}}, q_{y \mathrm{r}}=\frac{k_{y \mathrm{r}} \rho_{\mathrm{r}} h_{\mathrm{r}}}{\mu_{\mathrm{r}} y_{\mathrm{r}}}, q_{x \mathrm{r}}=\frac{S_{\mathrm{r}} x_{\mathrm{r}}}{t_{\mathrm{r}}}, q_{y \mathrm{r}}=\frac{S_{\mathrm{r}} y_{\mathrm{r}}}{t_{\mathrm{r}}} ; \\
& A_{\mathrm{r}}=S_{\mathrm{r}} x_{\mathrm{r}} y_{\mathrm{r}} .
\end{aligned}
$$

Note that if the soil is isotropic, then $k_{x}=k_{y}=k$. Isotropy and the middle two equations in Eq. (18) imply that $x_{\mathrm{r}}=y_{\mathrm{r}}$.

Combining the six independent equations requires six variables to be chosen arbitrarily. For example, if $x_{\mathrm{r}}, S_{\mathrm{r}}, \mu_{\mathrm{r}}$, $\rho_{\mathrm{r}}, W_{\mathrm{r}}$, and $t_{r}$ are chosen, then

$$
y_{\mathrm{r}}=x_{\mathrm{r}}, k_{\mathrm{m}}=k_{\mathrm{p}} \frac{S_{\mathrm{r}} x_{\mathrm{r}}^{2} \mu_{\mathrm{r}}}{h_{\mathrm{r}} t_{\mathrm{r}} \rho_{\mathrm{r}}}, h_{\mathrm{r}}=\frac{W_{\mathrm{r}} t_{\mathrm{r}}}{S_{\mathrm{r}}} .
$$

$q_{\mathrm{r}}$ and $A_{\mathrm{r}}$ are given in Eqs. (19) and (20), respectively. Further restrictions can be imposed on Eqs. (18), (19), and (20) such as similarity of the Reynolds, Froude, or Péclet numbers, similarly to the analysis in Sect. 2.2.

The modified inspectional analysis is very similar in application to the Lie scaling, producing similar results for the equations on the interior of the domain. This method is difficult to apply to problems where the parameters $k, \mu, \rho, S$, and $W$ vary as functions of space, time, or with respect to flow variables. The manner in which the variables must scale when they are non-constant is made clear through the Lie group theory. 


\subsection{An example comparing Lie and dimensional scaling: contaminant transport}

For a simple and straightforward example, consider a linear 1-D contaminant transport problem; see Bear and Buchlin (1991) for a detailed explanation. The contaminant has concentration $c$ moving with velocity $v$ and lumped dispersiondiffusion coefficient $D$. Assume that $v, D$ are constant values.

The concentration of the contaminant at location $(x, t)$ is given by $\partial c / \partial t+v \partial c / \partial x=D \partial^{2} c / \partial x^{2}$. This problem will first be analyzed using dimensional analysis and scaling, and then by Lie scaling.

Dimensional analysis can be applied very easily. The simplicity of the problem does not warrant the full Buckingham$\Pi$ theorem application. It is clear that the characteristic timescale is related to the velocity $v$ and the characteristic length scale $L: T=v t / L, X=x / L$. The non-dimensional concentration equation is

$$
\frac{\partial c}{\partial T}+\frac{\partial c}{\partial X}=\frac{D}{v L} \frac{\partial^{2} c}{\partial X^{2}} .
$$

The dimensionless quantity $\frac{v L}{D}$ is known as the Péclet number with characteristic length $L: P e_{L}$.

The non-dimensional equation indicates that the Péclet number has a profound effect on the dynamics of the system. For large values of $P e_{L}$, Eq. (21) resembles a linear advection equation. For small values of $P e_{L}$, the equation resembles the linear diffusion equation. When scaling this problem, it is desirable to keep the same Péclet number of the model as the prototype, given the importance of the $P e$ in the dynamics. It will be required that

$P e_{\mathrm{m}}=\frac{v_{\mathrm{m}} L_{\mathrm{m}}}{D_{\mathrm{m}}}=\frac{v_{\mathrm{p}} L_{\mathrm{p}}}{D_{\mathrm{p}}}=P e_{\mathrm{p}}$.

The Lie scaling approach begins with the direct scaling of all relevant variables and parameters. Following the notation above the ratios $x_{\mathrm{r}}=x_{\mathrm{m}} / x_{\mathrm{p}}$ and $t_{\mathrm{r}}=t_{\mathrm{m}} / t_{\mathrm{p}}, v_{\mathrm{r}}, c_{\mathrm{r}}$, and $D_{\mathrm{r}}$ are formed and a change in variable to the model variables is performed. In order for the equations to be invariant, the following set of equations for the ratio relationships must be satisfied:

$\frac{c_{\mathrm{r}}}{t_{\mathrm{r}}}=\frac{c_{\mathrm{r}} v_{\mathrm{r}}}{x_{\mathrm{r}}}=\frac{D_{\mathrm{r}} c_{\mathrm{r}}}{x_{\mathrm{r}}^{2}} \Rightarrow t_{\mathrm{r}}=\frac{x_{\mathrm{r}}}{v_{\mathrm{r}}}$ and $\frac{x_{\mathrm{r}} v_{\mathrm{r}}}{D_{\mathrm{r}}}=1$.

The results for the equations on the interior domain are identical to the conclusions of the dimensional analysis and dimensional scaling. In addition to Eq. (23), the Lie method requires the simultaneous scaling of the boundary conditions. Two common boundary conditions in contaminant transport are prescribed concentration and prescribed flux. Denoting $\mathcal{B}_{\mathrm{c}}$ as the prescribed concentration boundary and $\mathcal{B}_{\mathrm{f}}$ as the prescribed flux boundary, these boundary conditions are generally written as

$c(x, t)=g_{1}(x, t)$ on $\mathcal{B}_{\mathrm{c}}$,

(concentration) $c q-S D \frac{\partial c}{\partial x}=g_{2}(x, t)$ on $\mathcal{B}_{\mathrm{f}}$.

(flux)

$S$ is the porosity of the medium (= 1 if there is no porous matrix) and $q$ is the specific discharge at the boundary. $g_{1}$ and $g_{2}$ are the prescribed concentration and flux, respectively. A special case of the flux condition is on an impervious boundary. In this case $q \equiv 0$ and $g_{2} \equiv 0$; hence, $D \partial c / \partial x=0$ on $\mathcal{B}_{\mathrm{f}}$.

Invariance on the boundary requires that the functions $g_{1}(x, t)$ and $g_{2}(x, t)$ be self similar functions with respect to the scaled variables $x, t$. It was shown in Ibragimov (1995) that if $f\left(a x, a^{\beta} t\right)=a^{\gamma} f(x, t)$, then $f(x, t)=x^{\gamma} \Phi\left(t x^{-\beta}\right)$ for an arbitrary function $\Phi(\cdot)$. Changing variables in the boundary conditions and enforcing invariance lead to the requirements that

$c_{\mathrm{r}} q_{\mathrm{r}}=S_{\mathrm{r}} \frac{D_{\mathrm{r}} c_{\mathrm{r}}}{x_{\mathrm{r}}}=g_{2 \mathrm{r}} \Rightarrow q_{\mathrm{r}}=S_{\mathrm{r}} \frac{D_{\mathrm{r}}}{x_{\mathrm{r}}}$ and $c_{\mathrm{r}}=\frac{\left(g_{\mathrm{r}} / S_{\mathrm{r}}\right) x_{\mathrm{r}}}{D_{\mathrm{r}}}$.

Notice that $g_{\mathrm{r}} / S_{\mathrm{r}}$ is the velocity in the porous matrix at the boundary, so $\left(g_{\mathrm{r}} / S_{\mathrm{r}}\right) x_{\mathrm{r}} / D_{\mathrm{r}}$ is a Péclet number ratio on the boundary with characteristic length scaled as $x_{\mathrm{r}}$. In the case of an impervious boundary any scaling scheme preserves invariance on the boundary. For a prescribed concentration at the boundary, the scaling of the concentration must be the same as the scaling of the prescribed concentration; $c_{\mathrm{r}}=g_{1 \mathrm{r}}$.

Comparing the Lie scaling and dimensional scaling, it is seen that the results are the same for the equation on the interior of the flow domain. The differences between the methodologies stem from the treatment of the boundary by the Lie scaling. The conclusions from the boundary dictate that the concentration must scale in the same way as the prescribed concentration (either or both at the boundary or the initial concentration), and according to Eq. (24) simultaneously. The functional form of the prescribed flux and concentration functions $g_{1}(x, t)$ and $g_{2}(x, t)$ is restricted by the condition of self similarity as well.

The Lie scaling gives a complete picture of the requirements that must be satisfied in order for the full model to preserve the dynamics of the prototype boundary value problem. The other scaling methods focus on preserving a subset of dynamics, e.g., the dynamical similarity, the kinematic similarity, or invariance for the interior equations. The dimensional scaling methods do not consider parameter functions to be satisfying functional scaling relationships.

\section{Conclusions}

The three scaling techniques were described, applied, and compared. Each method will briefly be summarized below and comments made on each method's strengths and weaknesses.

The classical scaling method and most widely used technique is known as dimensional scaling. The basics of the method are an analysis through non-dimensionalization 
when equations describing the governing dynamics are known and application of the Vaschy-Buckingham theorem when governing equations are either not known or poorly understood.

The relative strengths of this method are its universal applicability, ease of application, and identification of the important non-dimensional parameter groups in a system and the effect they have on the solution process in the system. It is extremely useful to be able to apply the Vaschy-Buckingham theorem to analyze a problem about which very little is known. In cases where the closed form equations are not available to model dynamics, very basic information may be available on variables, parameters, and processes that contribute to a phenomenon. Gathering the dimensions for these quantities is enough to apply the $\Pi$ theorem and make judgements on modeling and scaling of such systems. In cases where governing equations are known and understood, dimensional analysis of the equations similar to the example in Sect. 3.1 can be used to derive the relevant dimensionless parameter groups and determine their effect on the system of equations.

The weakness of the classical dimensional scaling lies in the limited detail gained through an analysis. Generally, dimensional analysis is only applied to the dynamics in the interior of the domain and not to the boundary phenomena. This results in the neglect of influences from the boundary, which can be substantial in many problems. Dimensional scaling also overlooks the need for parameter functions or processes depending on variables that are being scaled to satisfy self similarity conditions in order to preserve the dynamics of the system. This is somewhat a strength and a weakness. It is a strength in that it gives the modeler a larger amount of freedom in creating the model. It is a weakness because it fails to identify the conditions in which a model system is truly a scaled version of the prototype, i.e., when a scaling transformation is invariant.

The modified inspectional analysis is very similar to the Lie scaling method both in development and in application. It is a somewhat intuitive version of the Lie scaling technique. It operates by examining the governing equations, applying a scaling transformation to the variables in the system, and enforcing invariance of the scaling transformation. Similarly to the dimensional scaling, this method does not explicitly consider that parameters or secondary processes that are functions of the scaled state variables of the system need to satisfy self similarity relationships. Rather, model processes can be chosen to have different dynamical properties than the prototype processes (Bear, 1972). For example, an aquifer with an anisotropic hydraulic conductivity can be modeled as an isotropic aquifer.

The Lie scaling technique is a powerful method based on a well-developed mathematical theory. In fact, the Lie scaling is an instance of a much larger class of invariant transformations which act on a system. In application, it involves development of the set of model variables by scaling all quantities in the prototype system, performing the change in variables in the prototype governing equations, and determining the precise scaling relationships by enforcing invariance on both the governing equations and boundary conditions. Note that the scaling transformation may also be found by applying the Lie algorithm, outlined above and described in detail in Bluman and Anco (2002), to determine the scaling transformation as well as the non-scaling invariant transformations.

The strength of the Lie scaling technique is that it gives a complete picture as to the set of conditions that a complete model must satisfy in order to preserve the dynamics of the prototype system. The precise nature of the dynamics to be preserved, e.g., kinematic similarity and dynamical similarity, can be explored and specified along with the general invariance of the transformation on the system of equations. It will not necessarily always be the case that a nontrivial scaling is possible which preserves all similarity relationships, but the simple fact that this can be unambiguously determined for the set of all possible invariant scaling transformations is itself fundamental. The Lie scaling approach requires that any known function or process that depends on scaled variables satisfy a self similarity relationship. Investigation of when functions will satisfy self similarity relationships, which gives insight into the applicable extent of scaling transformations and the regimes in which multi-scale models must be developed for a particular problem. Particularly for hydrological phenomena, investigations have concluded that certain medium and fluid parameters, such as the hydraulic conductivity, undergo fundamental changes in their distribution as the scale increases from the pore scale to the watershed scale (and above) (Kavvas, 1999; Meerschaert et al., 2013).

The Lie scaling considers the complete model, meaning the interior and boundary conditions, enforcing invariance in scaling for all regions in the problem. This ensures that the effects of the boundary conditions will be accounted for in preservation of the dynamics. It also provides a link between the flow problems in domains adjacent to the problem domain. For example, for the investigation of a scaled model for a subsurface saturated zone receiving recharge from an unsaturated zone, either the forcing function in the Dupuit approximating equations or the boundary condition in the full 3-D nonlinear problem will require self similarity of the flow in the unsaturated zone. This is important for considering coupling dynamics between different systems. Examples include seawater intrusion into the groundwater system and the interaction of regional climate models with groundwater.

The Lie scaling method requires the governing equation for both the interior and the boundaries to be known, and the functional scaling properties for any variable dependent parameters to be known as well. Models based on the Lie scaling must include medium and flow parameters that have identical (scaled) structure to the prototype. This may introduce technical difficulties in producing precisely scaled physical models in the laboratory. The scaling procedure must be ap- 
plied to problems individually. Any change in boundary conditions, initial conditions, flow, or medium parameters may significantly alter the scaling structure and existence of invariant transformations.

Lastly, the Lie scaling method is a special case of the more general method to classify the Lie group of transformations. The Lie group method systematically considers invariant changes of variables that make an equation integrable. While scaling is an important symmetry, other members may yield useful simplifications or insight into difficult problems and should be considered in future research.

Edited by: N. Romano

\section{References}

Barenblatt, G. I.: Scaling, self-similarity, and intermediate asymptotics, Cambridge University Press, New York, NY, 1996.

Bear, J.: Dynamics of fluids in porous media, American Elsevier, New York, 1972.

Bear, J. and Buchlin, J.-M. (Eds.): Modelling and Applications of Transport Phenomena in Porous Media, in: Theory and Applications of Transport in Porous Media, Kluwer Academic Publishers, Dordrecht; Boston, 5, XII, 381 pp., doi:10.1007/978-94-0112632-8, 1991.

Bertrand, J.: Sur l'homogeneite dans les formules de physique, Comptes Rendus, 86, 916-920, 1878.

Bluman, G. W. and Anco, S. C.: Symmetry and Integration Methods for Differential Equations, in: Applied Mathematical Sciences, Springer-Verlag, New York, 154, X, 422 pp., doi:10.1007/b97380, 2002.

Buckingham, E.: Notes on the method of dimensions LXXIX, The London, Edinburgh, and Dublin Philosophical Magazine and Journal of Science, 42, 696-719, 1921.

Carr, K., Ercan, A., and Kavvas, M. L.: Scaling and self-similarity of one-dimensional unsteady suspended sediment transport with emphasis on unscaled sediment material properties, J. Hydraul. Eng.-ASCE, 141, doi:10.1061/(ASCE)HY.1943-7900.0000994, 2015.

Carvallo, E.: Sur une similitude dans les fonctions des machines, J. Phys. Theor. Appl., 1, 209-212, doi:10.1051/jphystap:018920010020901, 1892.

Cayar, M. and Kavvas, M. L.: The average and ensemble variance behavior of unsteady, one-dimensional groundwater flow in unconfined, heterogeneous aquifers: an exact second-order model, Stoch. Env. Res. Risk A., 23, 947-956, doi:10.1007/s00477-0080263-1, 2009a.

Cayar, M. and Kavvas, M. L.: Symmetry in Nonlinear Hydrologic Dynamics Under Uncertainty: Ensemble Modeling of 2D Boussinesq Equation for Unsteady Flow in Heterogeneous Aquifers, J. Hydrol. Eng., 14, 1173-1184, doi:10.1061/(ASCE)HE.1943-5584.0000112, 2009b.

Dawdy, D., Griffis, V., and Gupta, V.: Regional flood-frequency analysis: How we got here and where we are going, J. Hydrol. Eng., 17, 953-959, doi:10.1061/(ASCE)HE.19435584.0000584, 2012.
Ercan, A., Kavvas, M. L., and Haltas, I.: Scaling and self-similarity in one-dimensional unsteady open channel flow, Hydrol. Process., 28, 2721-2737, 2014.

Fourier, J. B. J.: Théorie Analytique de la Chaleur, Cambridge Library Collection - Mathematics, Cambridge University Press, Cambridge, available from Cambridge Library Collection, doi:10.1017/CBO9780511693229, 2009.

Goldenfeld, N.: Lectures on phase transitions and the renormalization group, Advanced Book Program, Addison-Wesley, Reading, 1992.

Gupta, V. K. and Mesa, O. J.: Horton laws for hydraulic-geometric variables and their scaling exponents in self-similar Tokunaga river networks, Nonlin. Processes Geophys., 21, 1007-1025, doi:10.5194/npg-21-1007-2014, 2014.

Gupta, V., Mantilla, R., Troutman, B., Dawdy, D., and Krajewski, W.: Generalizing a nonlinear geophysical flood theory to medium-sized river networks, Geophys. Res. Lett., 37, L11402, doi:10.1029/2009GL041540, 2010.

Haltas, I. and Kavvas, M. L.: Scale Invariance and Self-Similarity in Hydrologic Processes in Space and TIme, J. Hydrol. Eng., 16, 1439-1449, 2011a.

Haltas, I. and Kavvas, M. L.: Scale invariance and self-similarity in kine- matic wave overland flow in space and time, Hydrol Process., 25, 3659-3665, $2011 \mathrm{~b}$.

Haltas, I. and Ulusoy, S.: Scaling and scale invariance of conservation laws in Reynolds transport theorem framework, Chaos, 25 , 075406, doi:10.1063/1.4917246, 2015.

Ibragimov, N. H.: CRC Handbook of Lie group analysis of differential equations, vol. 2, CRC Press, Boca Raton, Fl., 1995.

Kavvas, M.: On the coarse-graining of hydrologic processes with increasing scales, J. Hydrology, 217, 191-202, 1999.

Lie, S.: Theorie der transformationsgruppen, Teubner, Leipzig, Vol. 1, 1888

Logan, J. D.: Applied Mathematics: A Contemporary Approach, J. Wiley, New York, 1987.

Macagno, E. O.: Historico-critical review of dimensional analysis, J. Frankl. Inst., 292, 391-402, 1971.

Meerschaert, M., Dogan, M., Dam, R. V., Hyndman, D., and Benson, D.: Hydraulic conductivity fields: Gaussian or not?, Water Resour. Res., 49, 4730-4737, doi:10.1002/wrcr.20376, 2013.

Noether, E.: Invariante variationsprobleme, Nachrichten von der Gesellschaft der Wissenschaften zu Gottingen, mathematischphysikalische Klasse, 235-257, 1918.

Olver, P. J.: Applications of Lie groups to differential equations, in: Graduate Texts in Mathematics, Springer-Verlag, New York, 107, doi:10.1007/978-1-4684-0274-2, 1986.

Polsinelli, J. and Kavvas, M. L.: Scaling of the unconfined groundwater equations in heterogeneous aquifers, Hydrol. Process., doi:10.1002/hyp.10844, online first, 2016.

Riabouchinsky, D.: Methode des variables de dimension zero, et son application en aerodynamique, L'aerophile, 1, 407-408, 1911.

Riabouchinsky, D.: The principle of Similitude, Nature, 95, 644 644, doi:10.1038/095644b0, 1915.

Sedov, L. I.: Similarity and Dimensional Methods in Mechanics, 10th Edn., CRC Press, Boca Raton, FL, 1993.

Strutt Lord Rayleigh, J. W.: The theory of sound, Macmillan and co., London, Vol. 1, 1877-1878. 
Vafai, K. (Ed.): Handbook of Porous Media, 2nd Edn., CRC Press, Boca Raton, FL, 2005.

Vaschy, A.: Théorie de l'électricité: Exposé des phénomènes électriques et magnétiques fondé uniquement sur l'expérience et le raisonnement, Paris, Librairie polytechnique, Baudry et Cie, 1896.

Vaschy, A.: Sur les lois de similitude en physique, Annales telegraphiques, 19, 25-28, 1892.
White, F.: Fluid Mechanics, 7th Edn., McGraw-Hill, New York, 2011.

Yung, C. M., Verburg, K., and Baveye, P.: Group Classification and Symmetry Reductions of the Non-linear Diffusion-Convection Equation $u_{t}=\left(D(u) u_{x}\right)_{x}-K^{\prime}(u) u_{x}$, Int. J. Nonlinear Mech., 29, 273-278, 1994. 